\title{
Alometria reprodutiva de Podocnemis unifilis (Testudines: Podocnemididae) na várzea do baixo rio Amazonas, Santarém, Pará, Brasil
}

\author{
Marina T. Pignati ${ }^{1} \&$ Juarez C. B. Pezzuti ${ }^{2}$
}

1. Programa de Pós-Graduação em Zoologia, convênio Universidade Federal do Pará e Museu Paraense Emílio Goeldi, Secretaria do Programa de Pós-Graduação em Zoologia, Av. Perimetral,
1901/1907, Terra Firme, Caixa Postal 399, 66017-970, Belém, Pará, Brasil. (marinateofilo@yahoo.com.br)
2. Núcleo de Altos Estudos Amazônicos, Universidade Federal do Pará, Setor Profissional, Av. Perimetral, Guamá, 66075-650, Belém, Pará, Brasil. (juca@ufpa.br)

\begin{abstract}
Reproductive allometry of Podocnemis unifilis (Testudines: Podocnemididae) in the flooded forest portion of lower Amazon River, Santarém, Pará, Brazil. The body size of the organisms represents an important parameter, which may have consequences on their ecology, reproductive activities, evolution and development. Allometry relationship is the study of the size (or growth) of a body part in relation to the final size (or growth) of the organism. This study analyzed the allometric relationships between females of Podocnemis unifilis (Troschel, 1848), their eggs and hatchlings, and between nest features and the clutch in the flooded forest portion (várzea) of lower Amazon River, Santarém, state of Pará, Brazil. Podocnemis unifilis clutches' were monitored in the Tabuleiro da Água Preta during the reproductive cycle of 2009. The females found nesting were measured and the physical features of the nests were measured, and the latter were also marked with numbered stakes. The eggs and hatchlings of these nests were removed and counted, and their biometry was performed. The female parameters analyzed (straight-line carapace length and mass) were correlated with almost all the egg and hatchling variables, with the exception of the egg length and the hatchling mass. Future studies directed to the better understanding of how the environmental features influence nest features can be applied, being useful for more effective applications of management actions for the species.
\end{abstract}

KEYWORDS. Chelonian, reproduction, allometric relationships, clutch size, Amazon flooded forest.

RESUMO. O tamanho do corpo dos organismos representa um parâmetro importante, podendo gerar consequências na sua ecologia, atividades reprodutivas, evolução e desenvolvimento. Relação alométrica é o estudo do tamanho (ou do crescimento) de uma parte do corpo relacionado com o tamanho (ou crescimento) total do corpo do organismo. Este estudo analisou a relação alométrica entre as fêmeas de Podocnemis unifilis (Troschel, 1848) e seus ovos e filhotes e entre as características dos ninhos e a ninhada em uma área de várzea do baixo rio Amazonas, estado do Pará, Brasil. As ninhadas de P. unifilis foram monitoradas no Tabuleiro da Água Preta durante o período reprodutivo de 2009. As fêmeas encontradas desovando foram medidas e seus respectivos ninhos marcados com estacas numeradas e as características físicas mensuradas. Os ovos e filhotes destes ninhos foram retirados e contados, e tiveram seus dados biométricos tomados. Os parâmetros das fêmeas analisados (comprimento retilíneo da carapaça e massa) correlacionaram-se fortemente às variáveis dos ovos (variável x) e filhotes (variável y), exceto com o comprimento do ovo e com a massa do filhote. Estudos futuros direcionados para melhor compreensão de como as características ambientais influenciam nas ninhadas podem ser aplicados, sendo úteis no manejo da espécie.

PALAVRAS-CHAVE. Quelônios, reprodução, relações alométricas, tamanho da ninhada, várzea amazônica.

O tamanho do corpo dos organismos representa um parâmetro importante, inclusive para as espécies de quelônios, podendo gerar consequências na sua ecologia, atividades reprodutivas, evolução e desenvolvimento (Preston \& Ackerly, 2004). Para os répteis, estudos têm demonstrado que a história de vida e a fisiologia dos ninhos influenciam o tamanho, a forma e o número de ovos e filhotes produzidos (Smith \& Fretwell, 1974; IVERSON \& EWERT, 1991). Ao atingir a maturidade, as fêmeas de quelônios deixam de utilizar parte da energia disponível para o crescimento e começam a usá-la na reprodução. $\mathrm{O}$ esforço do investimento reprodutivo em um maior número de ovos é vantajoso pelo fato da maioria das espécies não apresentarem cuidado parental, com exceção de Kinosternon flavescens Agassiz, 1857 (Chelidae) (IVERson, 1990).

O tamanho da ninhada em muitas espécies de quelônios apresenta variação intra- e interpopulacional, com maiores fêmeas apresentando maiores tamanhos de ninhada (GibBons, 1982; Congdon \& GibBons, 1985). A relação entre o tamanho corporal da fêmea e o tamanho da ninhada em quelônios tem sido relacionada com muitos aspectos da ecologia comportamental e biologia reprodutiva (Peters, 1983).
Relação alométrica é o estudo do tamanho (ou do crescimento) de uma parte do corpo relacionado com o tamanho (ou crescimento) total do corpo do organismo (Peters, 1983). Atualmente, extensivas e detalhadas informações têm sido descobertas, sugerindo uma relação direta entre a massa corpórea do animal e seus processos fisiológicos como, por exemplo, o incremento na quantidade de ovos em relação ao aumento do tamanho do corpo em animais longevos e que apresentam crescimento corporal permanente (GiBBONS, 1987; LARRIERA et al., 2004), bem como o investimento de energia na reprodução para espécies que não adotam cuidados parentais (DoL'NIK, 2000).

A correlação entre número de ovos e tamanho do corpo é verificada em diferentes espécies de Testudines (Wilbur \& Morin, 1988). Dos estudos existentes com tartarugas de água doce, a maioria delas descreve as relações alométricas entre o comprimento retilíneo da carapaça e massa corpórea em fêmeas (Pough, 1980), comprimento linear do plastrão da fêmea e número de ovos (VAnzolini, 1977; GibBons et al., 1978; Vanzolini \& Gomes, 1979; Gibbons, 1982; Congdon \& GibBons, 1985; Dodd JR, 1997) e tamanho da câmara de ovos e largura do rastro da fêmea (GiBBONs, 1982; CONGDON 
\& Gibbons, 1985; Dodd JR, 1997; Valenzuela, 2001; Bonach et al., 2006). Thorbjarnarson (1996), King (2000) e VERDADE (2001) correlacionaram parâmetros de fêmeas (comprimento e massa total), de ovos (comprimento e massa dos ovos) da ninhada (tamanho, massa, comprimento e largura) para explicar a reprodução em crocodilianos.

Podocnemis unifilis (Troschel, 1848), localmente conhecida como tracajá, possui ampla distribuição na Bacia Amazônica e Tocantins-Araguaia, e suas fêmeas apresentam tamanho médio de $50 \mathrm{~cm}$ quando adultas (Pritchard \& Trebbau, 1984).

Este estudo objetiva analisar a relação alométrica entre as fêmeas de $P$. unifilis e seus ovos e filhotes, e também entre as características do microambiente dos ninhos e das ninhadas em uma área de várzea do baixo rio Amazonas, Estado do Pará, Brasil.

\section{MATERIAL E MÉTODOS}

O estudo foi desenvolvido no Tabuleiro da Água Preta, município de Santarém, nordeste do estado do Pará, Brasil, nas margens de um lago de várzea, no baixo rio Amazonas (209'12.5”S, 54³7'51.7’'W), próximo à confluência dos rios Tapajós e Amazonas (Fig. 1). O ecossistema de várzea amazônica consiste de uma área úmida periodicamente inundada (Ribeiro, 2007) e sua vegetação é composta principalmente por campos periodicamente alagados, embora localmente ocorram áreas de floresta. Os solos dessas áreas são aluviais, ricos em nutrientes e matéria orgânica depositados pelas inundações (RibeIro, 2007). A região de várzea amazônica está sob frequentes processos de erosão e deposição, criando bancos arenosos que destroem as margens e praias de rios e lagos, gerando fenômenos conhecidos localmente como "terras caídas" e "terras crescidas" (Irion et al., 1997).

O Tabuleiro da Água Preta está inserido em uma área de várzea sob influência da comunidade Água Preta, que tem na pesca sua principal atividade econômica. Os moradores realizam a proteção do tabuleiro buscando preservar a área de desova e a manutenção do estoque dos quelônios para caça e alimentação local na época da cheia. O tabuleiro é recoberto por uma vegetação gramínea e arbustiva caracterizada principalmente por Mimosa pigra ("juquiri"), Echinochloa pyramidalis (“canarãna") e Paspalum fasciculatum ("muri”).

As desovas de $P$. unifilis foram monitoradas durante o período reprodutivo de 2009 (21 de setembro a 27 de dezembro de 2009). Durante o intervalo de dias em que ocorreram as desovas ( 21 de setembro a 20 de outubro em 2009) a porção monitorada do tabuleiro (30 ha) foi percorrida à noite para localização das fêmeas. Ao encontrar fêmeas desovando, aguardou-se o completo processo de desova para então proceder a mensuração das fêmeas com auxílio de paquímetro $( \pm 1.000 \mathrm{~mm})$ e balança $( \pm 10.000 \mathrm{~g})$; cinco medidas foram tomadas: comprimento retilíneo da carapaça $(\mathrm{mm})$, largura da carapaça $(\mathrm{mm})$, comprimento do plastrão $(\mathrm{mm})$, largura da cabeça $(\mathrm{mm})$ e massa $(\mathrm{g})$. Os ninhos foram marcados com estacas numeradas, registrando-se a data de postura, a localização geográfica com a utilização de um GPS) e as seguintes variáveis: profundidade inicial do ninho (distância entre a superfície do ninho e o primeiro ovo, em $\mathrm{cm}$ ), distância do ninho à margem do rio $(\mathrm{m}) \mathrm{e}$ altura do ninho em relação ao nível do canal do Lago da Água Preta $(\mathrm{cm})$. A porcentagem de cobertura vegetal acima da superfície dos ninhos foi determinada com um esferodensiômetro Modelo C (Lemmon, 1956). Para mensurar a inclinação da superfície do substrato utilizado pelas fêmeas para desova foi utilizado um clinômetro. Os ovos destes ninhos foram retirados manualmente, contados e mensurados em comprimento e largura $(\mathrm{mm})$, utilizando um paquímetro $(0,1 \mathrm{~mm}$ de precisão), e pesados $(\mathrm{g})$ com auxílio de uma balança digital $(0,01$ g de precisão). Durante a eclosão dos filhotes, os ninhos foram inspecionados novamente. Após a absorção total do vitelo, os filhotes eclodidos encontrados ainda no interior dos ninhos foram submetidos à biometria adotando-se os procedimentos utilizados para as fêmeas, com os mesmos paquímetro e balança utilizados na medição dos ovos.

A correlação de Spearman foi usada para avaliar a independência entre as variáveis das fêmeas. Como as variáveis apresentaram correlações fortes $(\mathrm{r} \geq 0,9512$; $\mathrm{p}<0,0001)$, as variáveis comprimento retilíneo da
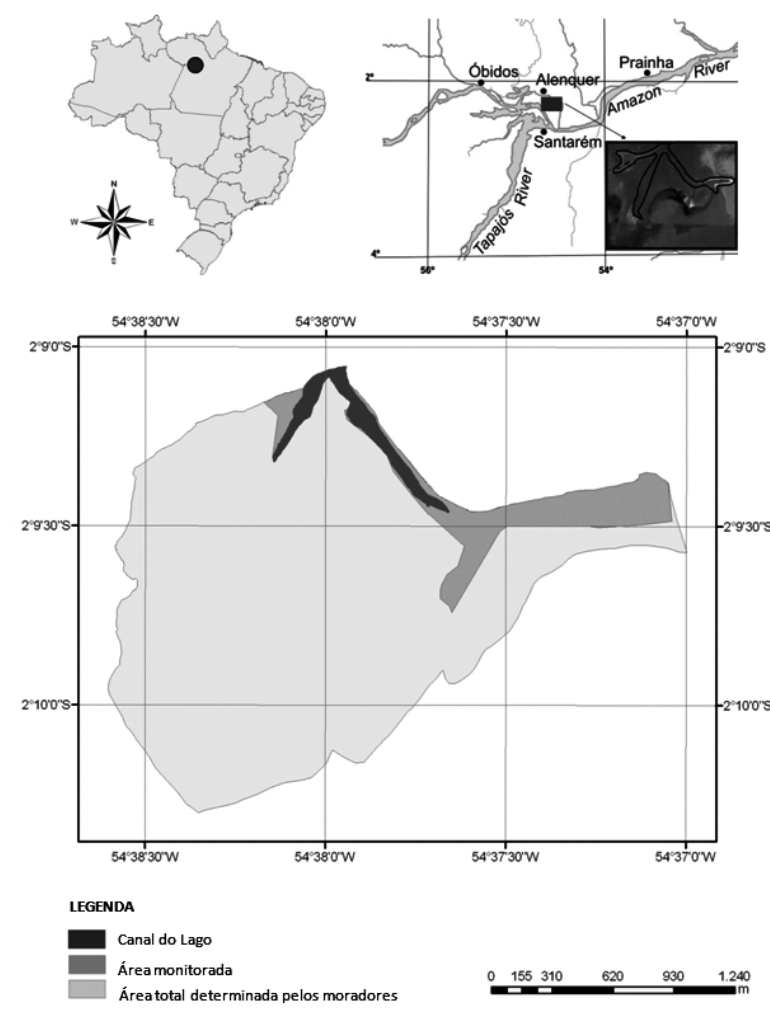

Fig. 1. Localização da área de estudo na comunidade Água Preta (quadrado), município de Santarém, Pará, Brasil; área monitorada na cor cinza média. 
carapaça e massa da fêmea foram selecionadas como expressão do corpo da fêmea. Os valores de cobertura vegetal foram transformados para arcoseno antes da realização das análises. Para verificar a relação existente entre as medidas das fêmeas e as variáveis dos ovos e filhotes, foram aplicados modelos de regressão linear simples, com o comprimento retilíneo da carapaça e a massa da fêmea como as variáveis independentes. Para verificar a relação existente entre as características dos ninhos analisadas (distância ao rio, altura, inclinação, profundidade inicial e porcentagem de cobertura vegetal) e as variáveis biométricas dos ovos e filhotes, foram aplicados modelos de regressão linear simples. A relação entre a massa de cada ovo e seu respectivo filhote foi determinada através da divisão da massa do ovo pela massa do filhote. Para verificar se as mesmas características dos ninhos acima detalhadas influenciam a relação entre a massa de cada ovo e seu respectivo filhote (variável dependente), foram aplicados modelos de regressão linear simples.

\section{RESULTADOS}

Foram encontradas e marcadas 11 fêmeas adultas, cujos ninhos foram monitorados durante todo o período de incubação. A primeira fêmea foi encontrada e marcada em 10 de outubro e a última em 20 de outubro de 2009.

As fêmeas apresentaram massa média de 6.045,45 g e comprimento retilíneo da carapaça médio de 378,82 $\mathrm{mm}$. Os ninhos apresentaram em média 29 ovos, estes com massa média de 22,9 g. A massa da ninhada representou em média $10,82 \%( \pm 1,80 ; 6,49-13,27 \%)$ da massa da fêmea. Os filhotes apresentaram em média 14,9 g e 39,60 mm de comprimento retilíneo de carapaça médio. Informações sobre a biometria das fêmeas, ovos e filhotes são mostradas na Tabela I.

Foram verificadas relações significativas e positivas entre a massa da fêmea e o tamanho da ninhada, massa da fêmea e a massa da ninhada, massa da fêmea e a massa média do ovo e entre a massa da fêmea e a largura média do ovo (Tab. II; Figs 2-5). Similarmente, obtivemos relações significativas e positivas entre a massa da fêmea e seguintes variáveis dos filhotes: comprimento retilíneo da carapaça, largura da carapaça e comprimento do plastrão (Tab. II; Figs 6-8). Não foram encontradas relações significativas entre a massa da fêmea e o comprimento do ovo e entre a massa da fêmea e a massa do filhote (Tab. II). Com relação ao tamanho corporal, representado pelo comprimento retilíneo da carapaça da fêmea, foram encontradas todas as mesmas relações significativas e positivas (Tab. II; Figs 9-15). Não foram registradas relações significativas entre o comprimento retilíneo da carapaça da fêmea e o comprimento do ovo e entre o comprimento retilíneo da carapaça da fêmea e a massa do filhote (Tab. II). Estes resultados mostram que quanto maior a fêmea, maiores serão seus ovos e filhotes.

As características dos ninhos avaliadas (distância ao rio, altura, inclinação, profundidade inicial e porcentagem de cobertura vegetal) não afetaram significativamente nenhuma das variáveis biométricas dos ovos e filhotes (P $>0,05)$. As características dos ninhos avaliadas também não afetaram significativamente a relação entre a massa de cada ovo e seu respectivo filhote $(\mathrm{P}>0,05)$.

Tab. I. Biometria de fêmeas, ovos e filhotes de Podocnemis unifilis (Troschel, 1848) do Tabuleiro da Água Preta, na várzea do baixo rio Amazonas, Santarém, estado do Pará, Brasil, em outubro de 2009 (DP, desvio padrão).

\begin{tabular}{lcccc}
\hline Variáveis & N & Média & Amplitude & DP \\
\hline Fêmeas & 11 & 378,82 & $325-414$ & 27,48 \\
$\quad$ Comprimento retilíneo da carapaça (mm) & 11 & 271,18 & $237-300$ & 20,21 \\
$\quad$ Largura da carapaça (mm) & 11 & 345,18 & $300-371$ & 22,34 \\
Comprimento do plastrão (mm) & 11 & 53,01 & $45-60$ & 4,35 \\
$\quad$ Largura da cabeça (mm) & 11 & $6.045,45$ & $4.000-8.250$ & $1.306,99$ \\
Massa (g) & & & & \\
Ovos & 11 & 29 & $18-36$ & 5,44 \\
Tamanho da ninhada (número de ovos) & 11 & 664 & $308,5-961,9$ & 207,77 \\
Massa da ninhada (g) & 319 & 22,9 & $15-59,90$ & 4,85 \\
Massa do ovo (g) & 319 & 43,40 & $35,40-67$ & 3,38 \\
Comprimento do ovo (mm) & 319 & 29,90 & $19,70-39,20$ & 2,61 \\
$\quad$ Largura do ovo (mm) & & & & 2,84 \\
Filhotes & 184 & 14,9 & $7,9-20,4$ & 2,54 \\
Massa do filhote (g) & 184 & 39,60 & $30,50-45,50$ & 3,17 \\
Comprimento retilíneo da carapaça (mm) & 184 & 34,01 & $10,80-38,90$ & 2,50 \\
Largura da carapaça (mm) & 183 & 38,32 & $30,40-43,10$ & \\
Comprimento do plastrão (mm) & & & \\
\hline
\end{tabular}


Tab. II. Alometria reprodutiva entre as variáveis dos ovos e filhotes e a massa da fêmea e entre as mesmas variáveis dos ovos e filhotes e o comprimento retilíneo da carapaça (CRC) da fêmea de Podocnemis unifilis (Troschel, 1848) na várzea do baixo rio Amazonas, Santarém, estado do Pará, Brasil, em outubro de 2009. Valores de P significativos em negrito (CRC, comprimento retilíneo da carapaça; LC, largura da carapaça; CP, comprimento do plastrão; $\mathrm{R}^{2}$ adj, $\mathrm{R}^{2}$ ajustado).

\begin{tabular}{|c|c|c|c|c|c|c|c|c|}
\hline \multirow{2}{*}{ Variáveis } & \multicolumn{4}{|c|}{ Massa da fêmea } & \multicolumn{4}{|c|}{ CRC da fêmea } \\
\hline & $\mathrm{N}$ & $\mathrm{F}$ & $\mathrm{P}$ & $\mathrm{R}^{2}$ adj & $\mathrm{N}$ & $\mathrm{F}$ & $\mathrm{P}$ & $\mathrm{R}^{2}$ adj \\
\hline Tamanho da ninhada & 11 & 8,2015 & 0,0180 & 0,4187 & 11 & 10,092 & 0,0110 & 0,4762 \\
\hline Massa da ninhada & & 41,7396 & 0,0003 & 0,8029 & & 31,7232 & 0,0005 & 0,7544 \\
\hline Massa do ovo & & 32,5574 & 0,0005 & 0,7594 & & 18,827 & 0,0022 & 0,6406 \\
\hline Comprimento do ovo & & 3,2213 & 0,1038 & 0,1818 & & 2,4016 & 0,1533 & 0,1229 \\
\hline Largura do ovo & & 39,8997 & 0,0003 & 0,7955 & & 35,1289 & 0,0004 & 0,7734 \\
\hline Massa do filhote & & 0,2702 & 0,6201 & $-0,0787$ & & 0,9611 & 0,6455 & $-0,0039$ \\
\hline CRC do filhote & & 10,5602 & 0,0099 & 0,4888 & & 12,0025 & 0,0072 & 0,5239 \\
\hline LC do filhote & & 6,961 & 0,0259 & 0,3735 & & 6,9324 & 0,0262 & 0,3723 \\
\hline CP do filhote & & 10,4616 & 0,0101 & 0,4862 & & 11,725 & 0,0076 & 0,5175 \\
\hline
\end{tabular}

\section{DISCUSSÃO}

Os dados de diversos estudos de biometria com P. unifilis variam de acordo com a sua distribuição geográfica. Os resultados da biometria de fêmeas, ovos e filhotes neste estudo são compatíveis com a literatura. VANZOLINI (1977) registrou para os rios Amazonas, Negro e Trombetas, em território brasileiro, fêmeas com média de $386 \mathrm{~mm}$ de comprimento da carapaça. Sмiтн (1979) mensurou 15 fêmeas no rio Amazonas, que apresentaram comprimento da carapaça médio de $355 \mathrm{~mm}$. Na Venezuela foram observadas fêmeas de $P$. unifilis com médias de $390 \mathrm{~mm}$ no rio Apure e $399 \mathrm{~mm}$ de comprimento da carapaça no rio Cinaruco (PRITCHARD \& TrebBau, 1984). A massa média das fêmeas capturadas na Colômbia foi $6.380 \pm 152 \mathrm{~g}(\mathrm{~N}=12)$ (Foote, 1978). De acordo com THORBJARNARSON et al. (1993), fêmeas de P. unifilis do Rio Capanaparo (Venezuela) apresentam média de $331 \mathrm{~mm}$ de comprimento da carapaça e 309 mm de comprimento do plastrão.

Foote (1978) obteve média de 27,4 ovos por ninhada de $P$. unifilis no rio Putumayo (na fronteira entre o Peru e a Colômbia). Pritchard \& Trebbau (1984) registraram para o rio Apure, ninhos com 24,4 ovos em média. ThorbJARNARSON et al. (1993) encontraram ninhadas no rio Capanaparo com média de 23,3 ovos, com média de $46 \pm 2 \mathrm{~mm}$ de comprimento, $31 \pm 1 \mathrm{~mm}$ de largura e massa de 26,5 $\pm 3,1 \mathrm{~g}$. Escalona \& Fa (1998) registraram no rio Nichare-Tawadu, na Venezuela, média de 20,1 ovos por ninho. Ninhos pesquisados na Reserva
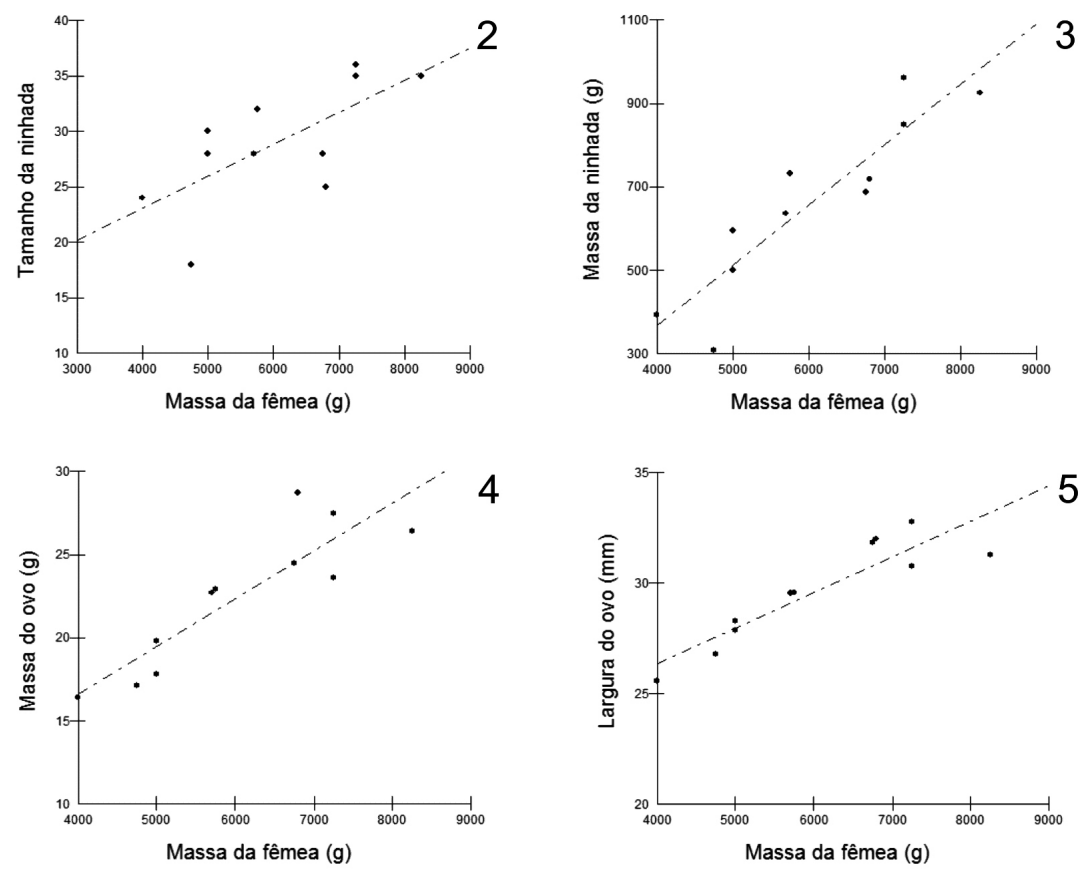

Figs 2-5. Relação entre as variáveis dos ovos e a massa da fêmea de Podocnemis unifilis (Troschel, 1848) na várzea do baixo rio Amazonas, Santarém, estado do Pará, Brasil: 2, tamanho da ninhada (número de ovos por ninho); 3, massa da ninhada; 4, massa do ovo; 5, largura do ovo. 

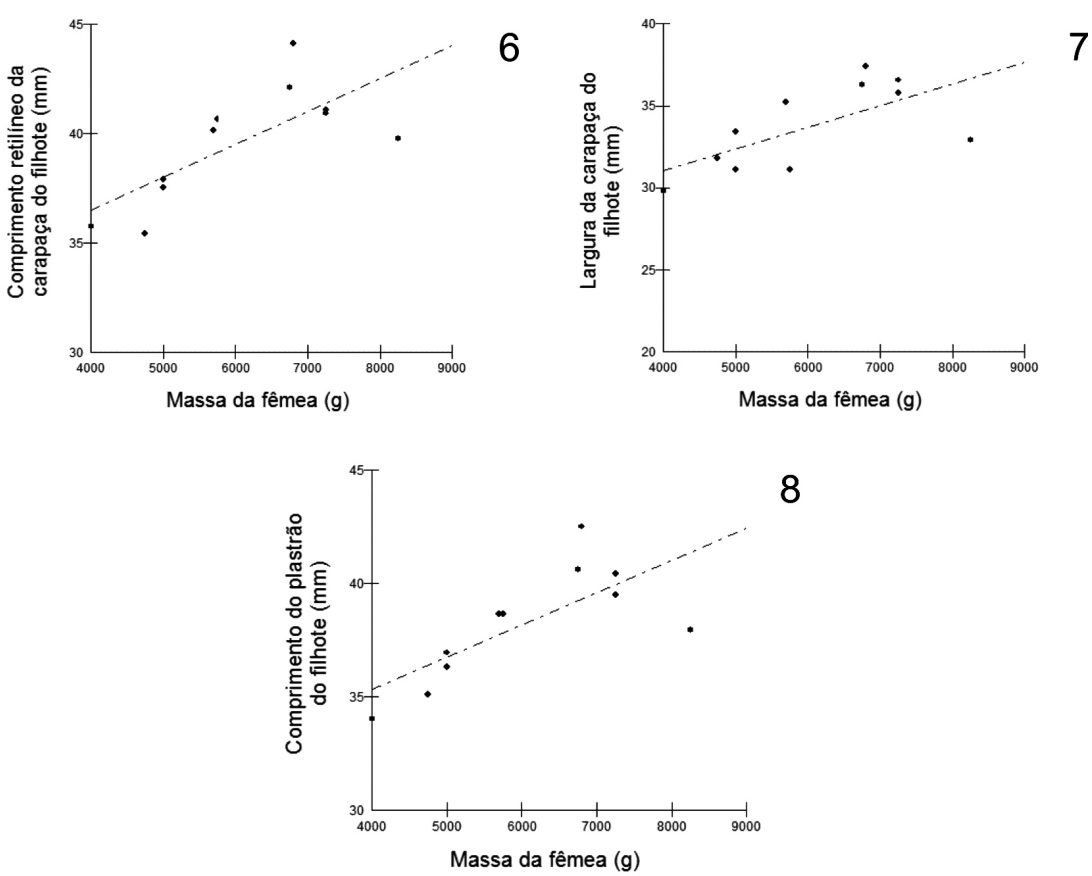

Figs 6-8. Relação entre as variáveis dos filhotes e a massa da fêmea de Podocnemis unifilis (Troschel, 1848) na várzea do baixo rio Amazonas, Santarém, estado do Pará, Brasil: 6, comprimento retilíneo da carapaça do filhote; 7, largura da carapaça do filhote; 8, comprimento do plastrão do filhote.
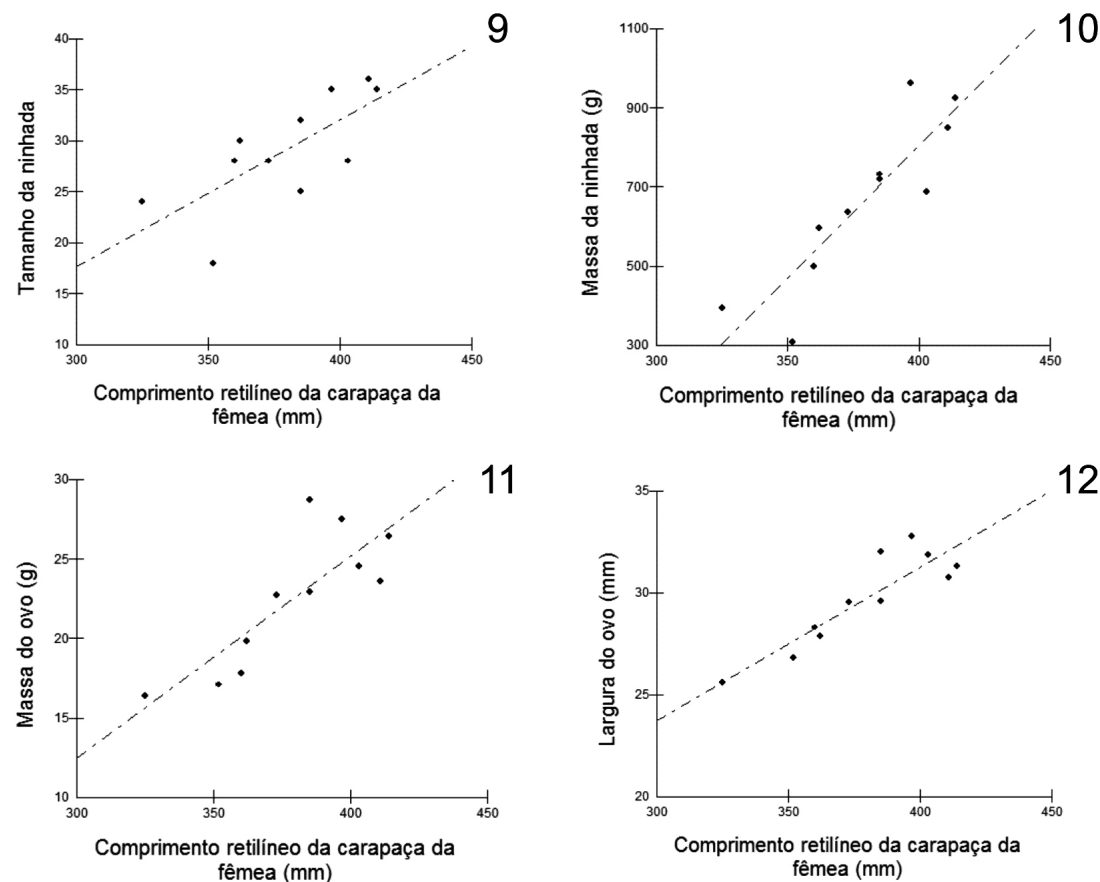

Figs 9-12. Relação entre as variáveis dos ovos e o comprimento retilíneo da carapaça da fêmea de Podocnemis unifilis (Troschel, 1848) na várzea do baixo rio Amazonas, Santarém, estado do Pará, Brasil: 9, tamanho da ninhada; 10, massa da ninhada; 11, massa do ovo; 12, largura do ovo.

de Desenvolvimento Sustentável Mamirauá, estado do Amazonas, apresentaram posturas mais numerosas, com média de 35,1 ovos por ninho; os ovos mediram em média 42,4 mm de comprimento, $30,7 \mathrm{~mm}$ e $23,2 \mathrm{~g}$ e os filhotes tiveram comprimento de carapaça de 43,1 $\mathrm{mm}$, largura de carapaça de $38,7 \mathrm{~mm}$ e massa de $16 \mathrm{~g}$ (FAchín-Terán \& Von Mülhen, 2003). Ferreira Júnior \& CASTRo (2010) registraram no rio Javaés, estado do
Goiás, tamanho da ninhada de $13,6 \pm 3,88$ ovos e 12,7 $\pm 3,86$ ovos, respectivamente, no ano de 2000 e 2001, resultado que mostra ninhadas com número de ovos bem reduzidos se comparado ao presente estudo. Segundo Fachín-Terán \& Von Mülhen (2003), nas áreas de várzea os ninhos apresentam maior número de ovos, pois são locais inundáveis que renovam seus nutrientes anualmente através dos rios que trazem sedimentos de 


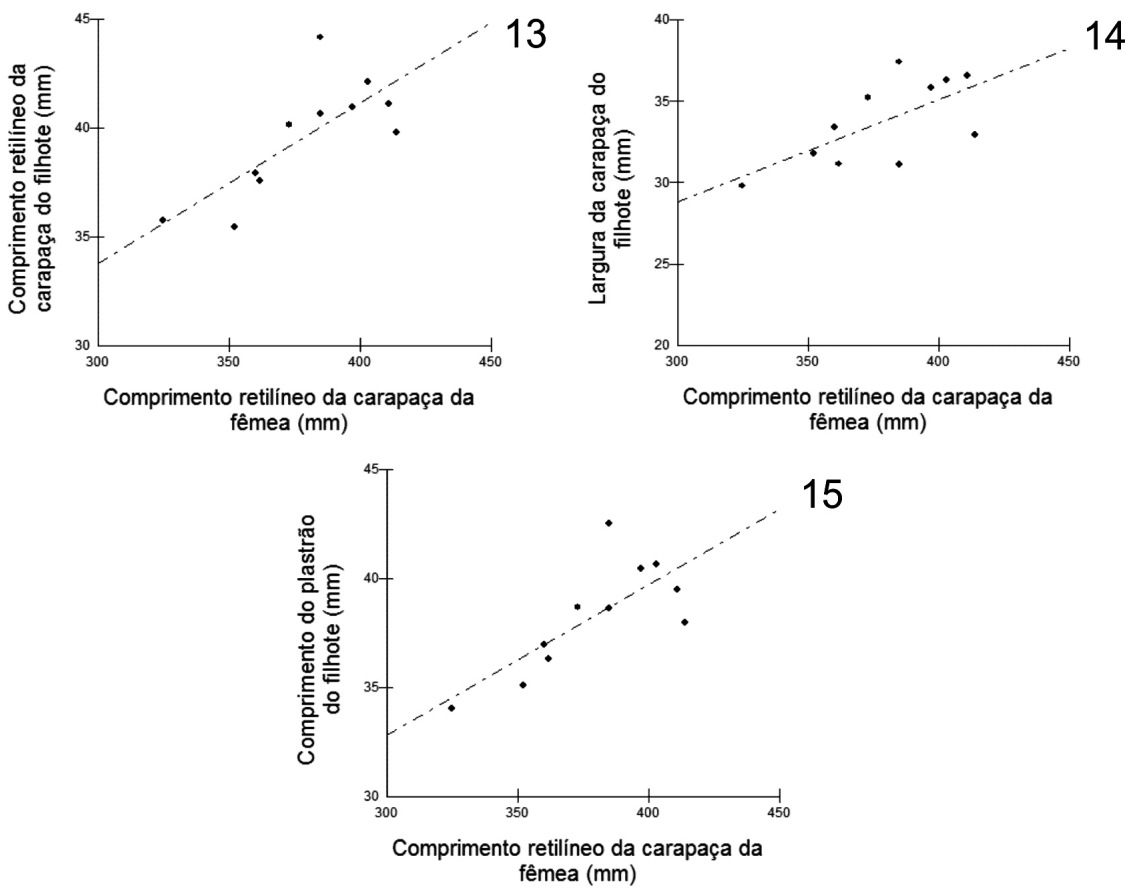

Figs 13-15. Relação entre as variáveis dos filhotes e o comprimento retilíneo da carapaça da fêmea de Podocnemis unifilis (Troschel, 1848) na várzea do baixo rio Amazonas, Santarém, estado do Pará, Brasil: 13, comprimento retilíneo da carapaça do filhote; 14, largura da carapaça do filhote; 15 , comprimento do plastrão do filhote.

origem andina, sendo muito produtivo em nutrientes disponíveis. Isto pode se refletir em melhor oferta de alimento em quantidade e qualidade, contribuindo tanto para o crescimento das fêmeas, que atingem desta forma tamanhos reprodutivos maiores, quanto para maior investimento na reprodução.

O tamanho do corpo tem sido base para muitos estudos de alometria reprodutiva (Peters, 1983). No presente estudo, foram utilizadas medidas do tamanho corporal da fêmea (representado pelo comprimento retilíneo da carapaça) e da massa da fêmea para estabelecer relações com as variáveis dos ovos e filhotes. Os parâmetros das fêmeas analisados correlacionaramse com quase todas as variáveis dos ovos e filhotes indicando que, apesar da variável massa da fêmea não ser extensivamente utilizada em estudos de alometria, esta também é uma boa preditora para relações alométricas, assim como o comprimento retilíneo da carapaça. Neste estudo obtivemos todas as relações significativas em comum, tanto para o parâmetro massa da fêmea quanto para o tamanho corporal em P. unifilis, indicando que estas medidas são influenciadas pelo aumento do tamanho do corpo e da massa das fêmeas. Segundo GibBons (1982), quanto maior o espécime, maior o tamanho médio da sua ninhada. Neste estudo, o mesmo padrão foi encontrado para $P$. unifilis, cujas fêmeas maiores depositaram ninhadas e ovos maiores, assim como a geração de filhotes com maiores tamanhos.

SoINI \& SoINI (1982) relataram que na população de $P$. unifilis na Reserva Nacional Pacaya-Samiria, no Peru, o volume e a largura dos ovos foram positivamente correlacionados com o tamanho da fêmea. O tamanho da ninhada é significativamente correlacionado com o tamanho da fêmea de $P$. vogli Müller, 1935 (Ramo, 1982). Além disso, Vanzolini \& GOMEs (1979) encontraram uma correlação positiva entre o comprimento do plastrão das fêmeas de $P$. sextuberculata Cornalia, 1849 e o tamanho da ninhada. Na Amazônia brasileira P. unifilis apresentou correlação positiva entre o tamanho da ninhada, o volume médio dos ovos, a forma de ovo e comprimento do plastrão da fêmea (Vanzolini, 1977). V. H. Cantarelli (dados inéditos) encontrou relações positivas entre a massa da fêmea e o tamanho da ninhada, massa da ninhada, comprimento retilíneo e largura da carapaça de filhotes de $P$. expansa Schweigger,1812 no rio Trombetas, estado do Pará. Nesta mesma área, o comprimento da carapaça das fêmeas de $P$. sextuberculata foi relacionado com a largura e volume do ovo e com a massa e o tamanho da ninhada, sendo este último relacionado também com a largura da carapaça da fêmea (HALler \& Rodrigues, 2006). De La Ossa \& Vogt (2011), em estudos com Peltocephalus dumerilianus (Schweigger, 1812) no estado do Amazonas, constatou que o volume total da ninhada foi relacionado ao comprimento da carapaça, comprimento do plastrão e altura do casco das fêmeas. Em estudos com P. expansa no rio Javaés, estado do Tocantins, o tamanho e a massa da ninhada foram relacionadas com o comprimento e largura da carapaça e a massa da fêmea, sendo a massa do ovo relacionada somente com o comprimento e a largura da carapaça (Portelinha et al., no prelo). Pezzuti et al. (2000) registraram correlação positiva entre a massa da fêmea de $P$. sextuberculata e a massa média dos ovos 
na Reserva Biológica do Abufari, estado do Amazonas. DoL'NIK (2000), ao estudar as relações alométricas entre diversos grupos de vertebrados, revelou várias relações específicas para o grupo dos répteis (quelônios) e a principal verificada foi que o tamanho da produção aumenta com o tamanho da fêmea.

Os resultados encontrados permitem afirmar que, para a população de $P$. unifilis estudada, não há correlação significativa entre as características dos ninhos e as características dos ovos e filhotes, resultado semelhante ao encontrado por PoRTelinha et al. (no prelo) para $P$. expansa no estado do Tocantins. BONACH et al. (2006) obtiveram relação significativa e entre o tamanho da ninhada e a largura da câmara de ovos e entre o tamanho da ninhada e a profundidade total do ninho de $P$. expansa nas praias dos rios Araguaia e Crixás-Açu, Goiás. Em estudos com $P$. expansa no médio rio Caquetá, na Amazônia colombiana, o tamanho da ninhada foi relacionado à profundidade da câmara de ovos (Valenzuela, 2001). Estes estudos apontam que, em termos quantitativos, certos aspectos da ninhada podem ser previstos através das características dos ninhos. Estes resultados, entretanto, possivelmente também estão correlacionados com o tamanho da fêmea, e não significam necessariamente que a largura e profundidade do ninho influenciam no desenvolvimento. É de se esperar que o tamanho da fềmea também influencie nas dimensões do ninho escavado, como largura e profundidade.

Os resultados do presente estudo demostram a importância do conhecimento das relações alométricas para P. unifilis na manutenção das populações, considerando principalmente a classe de tamanho das fêmeas que mais contribui: (1) em ninhadas com mais ovos, (2) em filhotes maiores ou ainda (3) em ninhadas com maiores chances de sobrevivência. Como as fêmeas não protegem seus ninhos após a desova, todo o investimento possível para que uma prole individual aumente sua chance de sobrevivência é necessário (Trivers, 1972). A vantagem adaptativa de produzir filhotes maiores ainda não está muito clara. Para filhotes de Trachemys scripta elegans (Wied, 1839) (Emydidae) a predação de filhotes foi dependente do tamanho dos filhotes, sendo que os maiores apresentaram uma redução da exposição à predação (JANZEN et al., 2000). A premissa que filhotes maiores têm melhores condições de sobreviver, denominada de hipótese do "maior é melhor" (bigger is better), é defendida por inúmeros autores (JANZEN 1993a,b; HASKELl et al., 1996; JANZEN et al., 2000). Em estudos com Chelydra serpentina (Linnaeus, 1758) (Chelydridae) em cativeiro, entretanto, BoByn \& BRoOKs (1994) mostraram que filhotes de tamanhos intermediários, com grandes reservas de vitelo, sobrevivem mais tempo e crescem mais rápido. Portanto, ainda não há um consenso entre sobre a hipótese do "maior é melhor". Estudos futuros direcionados para melhor compreensão de como as fatores ambientais influenciam as características das ninhadas podem ser aplicados, sendo úteis para aplicação mais efetiva de ações no manejo da espécie.
Agradecimentos. Os autores agradecem à Fundação de Amparo à Pesquisa do Estado do Pará (FAPESPA) pelo suporte financeiro e ao Instituto de Pesquisas Ambientais da Amazônia (IPAM) pelo suporte logístico durante a coleta de dados. Marina T. Pignati agradece a Priscila S. Miorando e Renata C. Vieira e aos moradores da comunidade Água Preta pela ajuda no trabalho de campo.

\section{REFERÊNCIAS BIBLIOGRÁFICAS}

Bobyn, M. L. \& Brooks, R. J. 1994. Incubation conditions as potential factors limiting the northern distribution of snapping turtles, Chelydra serpentina. Canadian Journal of Zoology 72:28-37.

Bonach, K.; Piña, C. I. \& Verdade, L. M. 2006. Allometry of reproduction of Podocnemis expansa in Southern Amazon basin. Amphibia-Reptilia 27:55-61.

Congdon, J. D. \& GibBons, J. W. 1985. Egg components and reproductive characteristics of turtles: relationships to body size. Herpetologica 41:194-205.

De La Ossa, J. L. V. \& Vogt, R. C. 2011. Ecologia populacional de Peltocephalus dumerilianus (Testudines, Podocnemididae) em dois tributários do rio Negro, Amazonas, Brasil. Interciencia 36:53-58.

DodD JR., C. K. 1997. Clutch size and frequency in Florida Box turtles (Terrapene carolina bauri): implications for conservation. Chelonian Conservation and Biology 2:370-377.

DoL'NIK, V. R. 2000. Allometry of Reproduction in Poikilotherm and Homoiotherm Vertebrates. Biology Bulletin 27:591-600.

Escalona, T. \& FA, J. E. 1998. Survival of nests of the terecay turtle (Podocnemis unifilis) in the Nichare - Tawadu Rivers, Venezuela. Journal of Zoology 244:303-312.

Fachín-Terán, A. \& Von Mülhen, M. 2003. Reproducción de la taricaya Podocnemis unifilis Troschel 1848 (Testudines: Podocnemididae) en la várzea del medio Solimões, Amazonas, Brasil. Ecologia Aplicada 2:125-132.

Ferreira Júnior, P. D. \& CASTRO, P. T. A. 2010. Nesting ecology of Podocnemis expansa (Schweigger, 1812) and Podocnemis unifilis (Troschel, 1848) (Testudines, Podocnemididae) in the Javaés River, Brazil. Brazilian Journal of Biology 70:85-94.

Fоoтe, R. W. 1978. Nesting of Podocnemis unifilis (Testudines: Pelomedusidae) in the Colombian Amazon. Herpetologica 34:333-339.

GibBons, J. W. 1982. Reproductive patterns in freshwater turtles. Herpetologica 38:222-227.

1987. Why do turtles live so long? BioScience 37:262-269.

Gibbons, J. W.; Greene, J. L. \& Schubauer, J. P. 1978. Variability in clutch size in aquatic chelonians. British Journal of Herpetology 6:13-14.

Haller, E. C. P. \& Rodrigues, M. T. 2006. Reproductive Biology of the Six-Tubercled Amazon River Turtle Podocnemis sextuberculata (Testudines: Podocnemididae), in the Biological Reserve of Rio Trombetas, Pará, Brazil. Chelonian Conservation and Biology 5:280-284.

Haskell, A.; Graham, T. E.; Griffin, C. R. \& Hestbeck, J. B. 1996. Size related survival of headstarted redbelly turtles (Pseudemys rubriventris) in Massachusetts. Journal of Herpetology 30:524527.

Irion, G.; JunK, W. J. \& Mello, J. A. S. N. 1997. The large Central Amazonian River floodplains near Manaus: geological, climatological, hydrological and geomorphological aspects. In: Junk, W. J. ed. The Central Amazon Floodplain: Ecology of a Pulsing System. Berlin, Springer. p. 23-46.

IVERSON, J. B. 1990. Nesting and parental care in the mud turtle, Kinosternon flavescens. Canadian Journal of Zoology 68:230233.

Iverson, J. B. \& Ewert, M. A. 1991. Physical characteristics of reptilian eggs and a comparasion with avian eggs. In: DeEming, D. C. \& Ferguson, M. W. J. eds. Egg Incubation: its effects on embryonic development in birds and reptilies. Cambridge, Cambridge University Press. p. 87-100.

JANZEN, F. J. 1993a. An experimental analysis of natural selection on body size of hatchling turtles. Ecology 74:332-341.

1993b. The influence of incubation temperature and family on eggs, embryos, and hatchlings of the smooth softshell turtle (Apalone mutica). Physiological Zoology 66:349-373. 
Janzen, F. J.; Tucker, J. K. \& Paukstis, G. L. 2000. Experimental analysis of an early life-history stage: selection on size of hatchling turtles. Ecology 81:2290-2304.

KING, R. B. 2000. Analyzing the relationship between clutch size and female body size in reptiles. Journal of Herpetology 34:148-150.

Larriera, A.; Piña, C. I.; Siroski, P. \& Verdade, L. M. 2004. Allometry of reproduction in wild broad-snouted caiman (Caiman latirostris). Journal of Herpetology 38:141-144.

Lemmon, P. E. 1956. A spherical densiometer for estimating forest overstory density. Forest Science 2:314-320.

Peters, R. H. 1983. The ecological implications of body size. Cambridge, Cambridge University Press. 329p.

Pezzuti, J. C. B.; Vogt, R. C.; Kemenes, A.; Félix-Silva, D.; Salvestrini, F. \& Lima, J. P. 2000. Nesting ecology of pelomedusid turtles in the Purus River, Amazonas, Brazil. In: Report of the 80th Annual Meeting of the American Society of Icthyologists and Herpetologists. La Paz, Universidad Autónoma de Baja California Sur. p. 294.

Portelinha, T. C. G.; Malvasio, A.; Piña, C. I. \& Bertoluci, J. Reproductive Allometry of Podocnemis expansa (Testudines, Podocnemididae) in Southern Brazilian Amazon. Journal of Herpetology (no prelo).

Pough, F. H. 1980. The advantages of ectothermy for tetrapods. The American Naturalist 115:92-112.

Preston, K. A. \& Ackerly, D. D. 2004. The evolution of allometry in modular organisms. In: Pigliucci, M. \& Preston, K. A. eds. Phenotypic Integration: Studying the Ecology and Evolution of Complex Phenotypes. New York, Oxford University Press. p. 80-106.

Pritchard, P. C. H. \& Trebbau, P. 1984. Family Pelomedusidae Cope, 1868. In: Pritchard, P.C.H. \& Trebbau, P. eds. The Turtles of Venezuela. Athens, Society for the Study of Amphibians and Reptiles. p. 33-73.
Ramo, C. 1982. Biología del galapago (Podocnemis vogli Muller, $1935)$ en el Hato El Frio. Acta Vertebrata 9:1-161.

Ribeiro, N. V. 2007. Atlas da várzea: Amazônia Brasil. Manaus, Ibama. 132p.

Smith, C. C. \& Fretwell, S. D. 1974. The optimal balance between size and number of offspring. American Naturalist 108:499-506.

Smith, N. J. H. 1979. Quelônios aquáticos da Amazônia: um recurso ameaçado. Acta Amazonica 9:87-97.

SoInI, P. \& Sorni, M. 1982. Ecología reproductiva de la taricaya (Podocnemis unifilis) y sus implicaciones en el manejo de la especie. Reporte Pacaya-Samiria 9:100-128.

ThORBJARnARson, J. B. 1996. Reproductive characteristics of the Order Crocodylia. Herpetologica 52:8-24

Thorbjarnarson, J. B.; Perez, N. \& Escalona, T. 1993. Nesting of Podocnemis unifilis in the Capanaparo River, Venezuela. Journal of Herpetology 27:344-347.

Trivers, R. L. 1972. Parental investment and sexual selection. In: Cambell, B. G. ed. Sexual Selection and the Descent of Man. New Jersey, Transection Publishers. p. 136-179.

Valenzuela, N. 2001. Constant, shift and natural temperature effects on sex determination in Podocnemis expansa turtles. Ecology 82:3010-3024.

VAnZolini, P. E. 1977. A brief biometrical note on the reproductive biology of some South American Podocnemis (Testudines, Pelomedusidae). Papéis Avulsos de Zoologia 31:79-102.

Vanzolini, P. E. \& Gomes, N. 1979. A note of the biometry and reproduction of Podocnemis sextuberculata. Papéis Avulsos de Zoologia 32:277-290.

VERDADE, L. M. 2001. Allometry of reproduction in broad-snouted caiman (Caiman latirostris). Brazilian Journal of Biology 61:431-435.

Wilbur, H. M. \& Morin, P. J. 1988. Life history evolution in turtles. In: Gans, C. \& Huey, R. B. eds. The biology of the Reptilia: defense and life history. New York, Alan R. Liss. p. 387-439. 